\title{
Con la lengua como principal herramienta: una aproximación al espectáculo Fiddler on the Roof en ídish
}

Fecha de recepción: 22/08/2020. Fecha de aceptación:11/09/2020

\begin{abstract}
Resumen
En 2018 se estrenó en Nueva York la obra Fiddler on the Roof/A fidler afn dakh (en español: El violinista en el tejado), versión en ídish del famoso musical de Broadway basado en las historias del escritor judío Sholem Aleichem. El espectáculo fue llevado a escena por el Folksbine, una de las compañías de teatro ídish más antiguas del mundo y, debido a su gran éxito, se mantuvo en cartel en una sala del circuito off-Broadway hasta principios del 2020. Este trabajo indaga en este fenómeno a partir de un análisis de los elementos de la puesta en escena, preguntándose especialmente por la incidencia y el papel que la utilización del ídish tuvo en el impacto de la obra en el público.
\end{abstract}

Palabras claves: Fiddler on the Roof, idish, Nueva York, teatro judío, Aleichem.

\section{Language as Main Tool: an Approach to Fiddler on the Roof in Yiddish}

\begin{abstract}
In 2018, Fiddler on the Roof/A fidler afn dakh-a version of the famous Broadway musical based on the stories of the Yiddish writer Sholem Aleichem- premiered in New York. The show was produced by the Folksbine, one of the oldest Yiddish theatre companies in the world. Due to its great success, the play ran in an off-Broadway theatre until the beginning of 2020. This essay analyzes this phenomenon by exploring the different elements of the show, focusing especially on the role that the use of the Yiddish language had in its impact on the audience.
\end{abstract}


Nueva York, Estados Unidos. En un teatro del circuito off-Broadway, ocho veces por semana, sucede una maravilla de maravillas: una compañía teatral realiza funciones de la obra Fiddler on the Roof/A fidler afn dakh (en español: El violinista en el tejado) en idioma ídish, a sala llena. En una vuelta que lo lleva nuevamente al punto de inicio, el famoso musical basado en las historias del autor judío Sholem Aleichem (18591916) vuelve al idioma en el que fueron escritas originariamente. O, como dice en el programa de mano del espectáculo, al idioma en el que hablaban Tevye y su familia: el ídish, la lengua utilizada por la mayor parte de los judíos de Europa del Este y Rusia hasta la Segunda Guerra Mundial.

Esta nueva versión del musical de Broadway es producida por el National Yiddish Theatre Folksbiene (NYTF), la compañía más antigua de teatro en ídish que aún funciona de manera regular en Nueva York. Desde finales del siglo XIX y especialmente en las primeras décadas del XX, Nueva York fue la capital indiscutida del teatro ídish mundial. El Folksbine (Teatro del Pueblo) se fundó en 1915, en el contexto de este gran auge, bajo los auspicios del Arbeter Ring (El Círculo Obrero), y se convirtió en una de las más importantes compañías de teatro ídish del mundo y en una de las pocas que continúa en funcionamiento hasta hoy. En este sentido, el NYTF asegura la permanencia del ídish en los escenarios de la ciudad y mantiene viva así una tradición teatral que contribuyó a darle forma al campo teatral de Nueva York. ${ }^{1}$

También las historias de Tevye y su familia cuentan con una larga historia de representaciones en el escenario. Ya el propio Scholem Aleichem realizó una adaptación teatral de sus escritos con el título Tevye, dermilkhiker (Tevye, el lechero), popularizada en los escenarios judíos por el gran actor y director Maurice Schwartz, creador del Yiddish Art Theatre, quizás el teatro de arte judío más importante de la época. La obra fue incluso llevada a la gran pantalla en 1939, protagonizada por el mismo Schwartz. La producción de cine en ídish estuvo estrechamente vinculada al teatro, dado que por lo general eran las estrellas teatrales quienes protagonizaban los films llevando muchas veces al cine sus propias producciones escénicas.

Posteriormente, el dramaturgo norteamericano Arnold Perl adaptó, en 1957, esos mismo textos al escenario, esta vez en inglés, en su obra teatral Tevye y sus hijas. En otra interesante vuelta al origen, esta obra fue representada en Buenos Aires por el Teatro IFT en 1959 en una versión al ídish realizada por Ioel Lincovsky, padre de la famosa actriz judeo-argentina Cipe Lincovsky. Es en este texto dramático en el que se basó el musical de Stein, Bock y Harnick estrenado en 1964, que popularizó la figura de Tevye a lo largo de Estados Unidos y del mundo. El musical fue también llevado al cine en 1971 dirigido por Norman Jewison. Esta obra presenta una serie de cambios sustanciales con respecto a la historia original de Sholem Aleichem. Por ejemplo, en el musical de Broadway, Tevye y su familia emigran a América y no ya a Palestina, cambio que permitió que el público norteamericano pudiera establecer una conexión mayor con la historia. ${ }^{2}$

El espectáculo del NYTF se estrenó en julio de 2018 en el Museum of Jewish Heritage, dirigida por Joel Grey, actor y director de amplia trayectoria en el teatro musical y el cine. Debido a la buena recepción de las representaciones entre el público y la crítica, en 2019 la producción pasó a un teatro del off-Broadway donde continuó representándose con gran éxito hasta principios de 2020. La traducción al ídish utilizada en esta versión fue realizada por Shraga Friedman y proviene de una producción israelí de la obra realizada en 1965, es decir, un año después del estreno del musical original en Broadway. Es importante señalar que se trata de

1 Para mayor desarrollo sobre la historia del teatro ídish en Nueva York ver: Nahshon, E. (2016); Sandrow, N. (1996).

2 Para una historia acerca de la génesis de Fiddler on the roof ver: Isenberg, B. (2014); Solomon, A. (2013). 
una traducción no estrictamente literal palabra por palabra, sino interpretativa, ya que en muchos casos realiza una adaptación de los conceptos del inglés al ídish. Un ejemplo muy claro de esta operación la encontramos en la traducción de la famosa canción interpretada por Tevye "If I were a rich man" "Si yo fuera rico") que en esta versión se transforma en "Ven ikh bin a Rothschild", una expresión popular en ídish que hace referencia a la acaudalada familia de banqueros judíos y que es, a su vez, tomada de otra historia de Sholem Aleichem. En este sentido, si uno observa los subtítulos en inglés que se proyectan en el escenario y que corresponden a los parlamentos de la obra original, pueden notarse numerosas diferencias con respecto a lo expresado por los personajes en ídish. La traducción mantiene así el sentido del texto, pero, al tomar expresiones y figuras del lenguaje propias del universo cultural ídish, le otorga a los diálogos una dimensión simbólica extra y una autenticidad mayor.

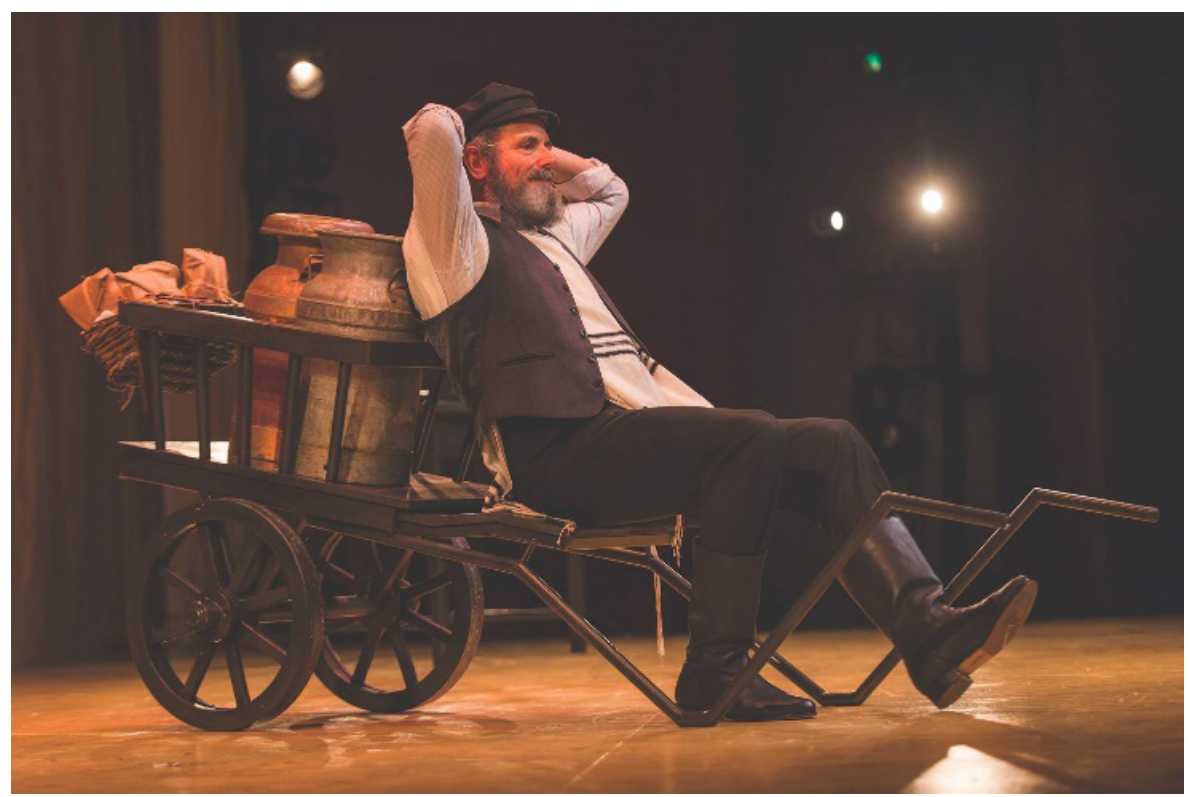

Foto: Victor Nechay - properpix.com

Por otra parte, es interesante detenernos en el hecho de que se trata de un elenco compuesto en su mayoría por actores y actrices que no hablan el ídish y que han tenido que aprenderlo fonéticamente para la representación, una práctica habitual en la ópera, pero no así en el teatro. Sin embargo, a pesar de las dificultades que esto supone, el elenco logra otorgarle vitalidad a un idioma desconocido para gran parte del público, y transmitir sentidos y emociones, aún sin la ayuda de los subtítulos. En primer lugar, consideramos que esto se debe a que la sonoridad del ídish posee una carga simbólica y afectiva que ayuda a consolidar el ambiente de autenticidad de la obra. De esta forma, la lengua en la que se realiza la representación no resulta un obstáculo para el disfrute del espectáculo, como podría pensarse en un primer momento, sino que constituye uno de los componentes principales de su éxito. En este sentido, la obra realiza una operación contraria a la que las compañías teatrales judías llevaban adelante en el período de auge del teatro ídish, especialmente en el período de entreguerras, cuando este teatro comienza a atraer a una numerosa cantidad de espectadores no judíos que no comprendían la lengua utilizada en el escenario. En ese entonces, mucho antes de la existencia de los subtítulos, los directores judíos se veían obligados a desarrollar aspectos del lenguaje teatral que pudieran transmitir sentimientos e ideas con independencia de la comprensión de los diálogos. Como ha señalado Debra Caplan (2018), los teatros judíos de arte 
necesitaron desplegar una poética visualmente atractiva para mantener la atención del público que no era ídish parlante. La barrera idiomática de la audiencia se convirtió así en un estímulo para los artistas del teatro ídish para llevar adelante una mayor investigación en cuanto a la plástica de la escena, al trabajo del actor con su cuerpo y a la transmisión de emociones por vía no verbales. Lo mismo sucedió con respecto a los recursos de puesta en escena, en los cuales los directores judíos se apoyaban en mayor medida que otros para transmitir más claramente las ideas y emociones de la obra.

Por el contrario, Fiddler on the roof in Yiddish, como fue promocionada esta versión del musical, pone el acento justamente en su particularidad idiomática, ya desde su mismo nombre. Lejos del despliegue técnico y visual que caracterizó a las producciones de los teatros judíos de arte durante la primera mitad del siglo XX, la puesta del NYTF reduce los componentes escenográficos al mínimo llevando todo el peso del realismo a la lengua. Los decorados, por ejemplo, son sumamente sencillos: el escenario se encuentra cubierto por una serie de telones de papel de color amarronado que no buscan representar al pueblito de Anatevka de manera naturalista, sino mostrar simbólicamente la estabilidad inicial de la vida tradicional judía que se verá sacudida a lo largo de la obra. Por ese motivo, el elemento principal del diseño escenográfico es la palabra Toyre (Torá), escrita con grandes letras en el centro del fondo de papel coronando todas las acciones de los personajes ya que, como dice Tevye en su parlamento inicial, lo que rige la vida judía en Anatevka es justamente la "traditsye", la tradición basada en las enseñanzas de la Torá. Es así que cuando, en la escena del pogromo, los cosacos rompen el telón de papel con la palabra "Toyre" el público se sacude en sus asientos, puesto que ese gesto es suficiente para sentir la violencia perpetrada contra los judíos. Lo mismo sucede en la escena del bar en la que judíos y cosacos bailan juntos, donde la tensión entre ellos se expresa por medio de los pasos de danza violentos y amenazantes de los cosacos. La puesta en escena se construye así a partir de una gran simplicidad, que sin embargo logra otorgarle a los elementos escénicos una fuerte profundidad simbólica. El espectáculo consigue de esa forma transmitir con recursos simples y concretos problemáticas sumamente complejas, tales como la violencia antisemita y la precariedad de la vida judía en el Imperio Zarista, así como también la capacidad de resiliencia de los judíos, expresada en la reparación del telón de papel con la palabra Toyre, que aparece nuevamente unida en las escenas siguientes al pogromo.

De esta forma, los diferentes espacios en los que transcurre la obra se van construyendo con una gran economía de recursos. Por ejemplo, para marcar el ingreso a la casa de Tevye y su familia alcanza con el gesto de besar la mezuzá -el rollo de pergamino con versículos de la Torá que se coloca en el marco de las puertas de las casas judíaspara indicarle al espectador que nos encontramos en el espacio del hogar. En este sentido, la obra apela a la competencia de la audiencia para reconocer los elementos y poder interpretar su significado. Lo mismo sucede en el resto de las escenas, donde con algunos pocos, pero significativos, objetos de utilería se nos informa acerca de la situación dramática: el famoso carro lechero de Tevye, un par de mesas y sillas para las escenas en el bar, etc.

Asimismo, el diseño del vestuario posee también una sustancial carga simbólica. En primer lugar, todos los personajes visten ropas que se encuentran en la misma gama de colores, principalmente blanco, negro y gris. Esto es utilizado para expresar la vida homogénea que llevan los habitantes del pueblo y, a su vez, para ir marcando los cambios que se van produciendo en ellos y que se expresan a través de su vestuario. Es así que las hijas de Tevye (Tsaytl, Hodl y Khave) comienzan a vestirse con otros colores (azul, amarillo y rojo, respectivamente) cuando empiezan a tomar decisiones propias que se alejan de lo que indica la tradición, ya que se aventuran a elegir a sus 
propios maridos guiadas por el amor y no por lo que rigen las costumbres heredadas. Por otro lado, la ropa sirve también para marcar la distancia que separa a los personajes judíos de los cosacos, que en oposición a ellos, visten con colores rojos. Por este motivo, cuando Khave se enamora del ruso Fyedke, comienza a llevar una prenda roja en su vestimenta.

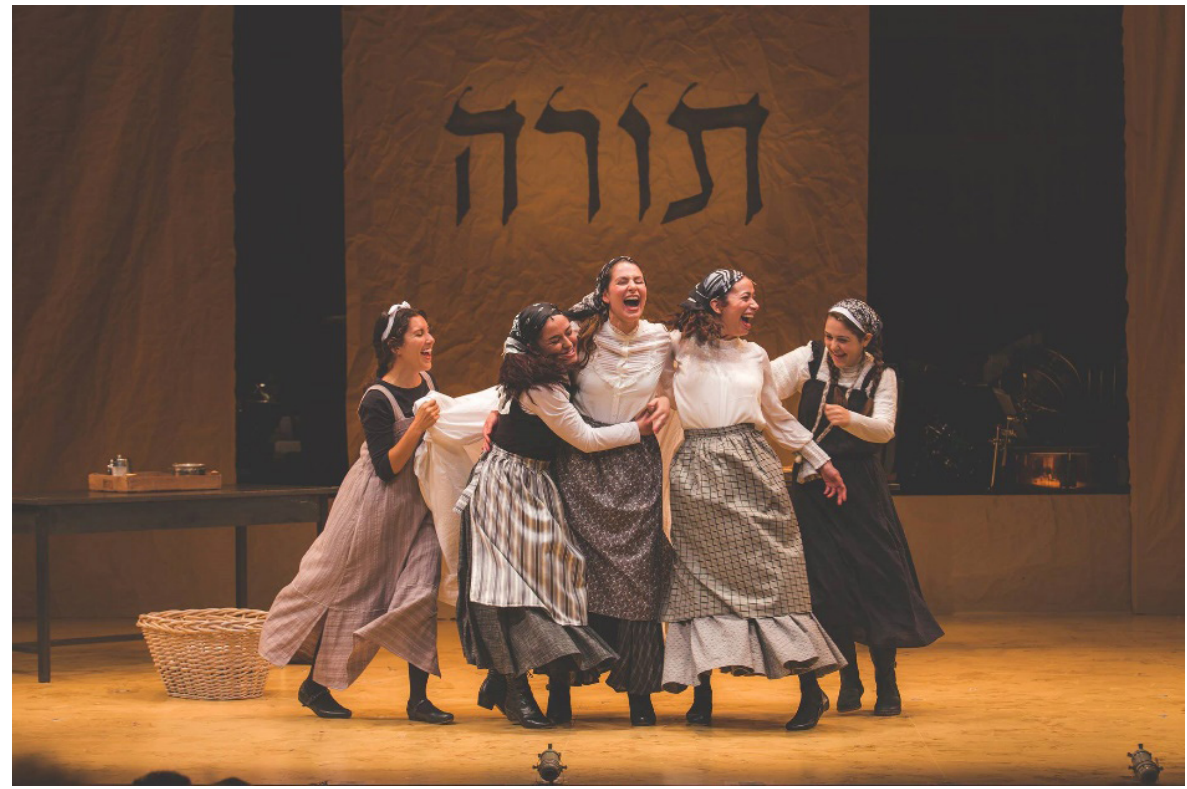

Foto: Victor Nechay - properpix.com

Estos recursos simples, pero fuertemente simbólicos, sumados al uso del ídish que le otorga un gran efecto de realidad a la puesta, nos permiten introducirnos en el universo judío de Anatevka e ir viviendo junto a los personajes su proceso de cambio. En este sentido, es justamente porque los personajes hablan en ídish que la puesta en escena puede apoyarse en una escasa escenografía y en algunos pocos elementos de utilería, sin necesidad de introducir detalles superfluos o procedimientos naturalistas. Como hemos postulado, la sonoridad de la lengua, más allá de su significado, contribuye a crear tanto una sensación de autenticidad como de intimidad con los espectadores, especialmente aquellos para quienes la lengua ídish posee una carga familiar y emocional propia. En las entrevistas a los integrantes de la obra, abundan las anécdotas respecto a los espectadores ídish-parlantes o descendientes de padres o abuelos ídish-parlantes que se acercaron emocionados a agradecerles a los actores y actrices por permitirles escuchar el idioma de su infancia en el escenario.

Por último, encontramos un elemento más que permite comprender el éxito de esta nueva puesta de Fiddler on the roof: el actual contexto sociopolítico de los Estados Unidos. En los últimos años, el país ha experimentado una oleada creciente de xenofobia, racismo y antisemitismo, en la cual discursos como los del Ku Klux Klan han vuelto a ganar terreno en la esfera pública. En este nuevo escenario sociopolítico, la historia de los judíos de Anatevka adquiere una resonancia mayor, al interpelar a las minorías que en la actualidad enfrentan persecuciones, exilios y migraciones forzosas. $Y$, en este sentido, el final de la obra, que presenta a los Estados Unidos como el lugar al que los personajes se dirigen en busca de libertad y de una vida mejor, nos incita a preguntarnos si Tevye y su familia podrían encontrar esa nueva oportunidad en el Estados Unidos al que llegarían hoy. 


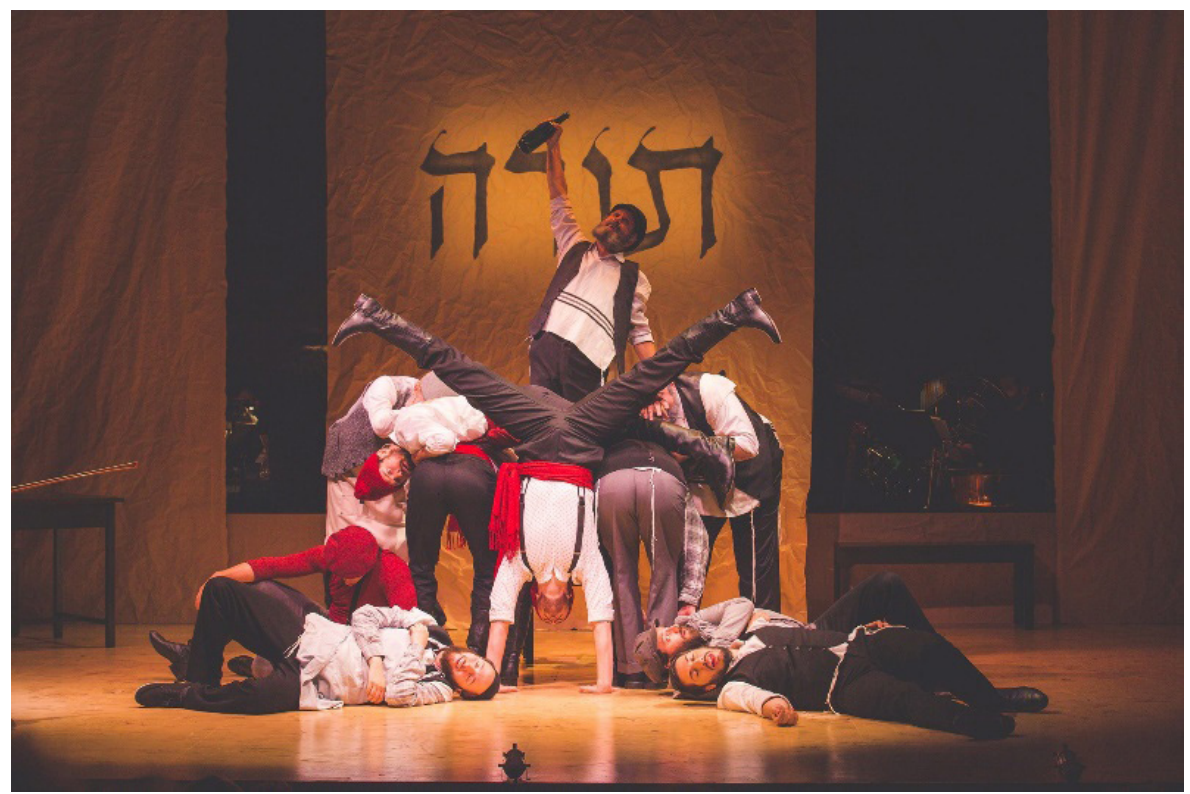

Foto: Victor Nechay - properpix.com

\section{Ficha técnico-artística}

Estrenada en Nueva York en julio de 2018 realizó funciones en el Museum ofjewish Heritage y en Stage 42 hasta enero de 2020.

$\begin{array}{ll}\text { Libro: } & \text { Joseph Stein } \\ \text { Música: } & \text { Jerry Bock } \\ \text { Letras de canciones: } & \text { Sheldon Harnick } \\ \text { Escenografía: } & \text { Beowulf Boritt } \\ \text { Vestuario: } & \text { Ann Hould-Ward } \\ \text { lluminación: } & \text { Peter Kaczorowski } \\ \text { Sonido: } & \text { Dan Moses Schreier } \\ \text { Coreografía: } & \text { Jerome Robbins } \\ \text { Dirección musical: } & \text { Zalmen Mlotek } \\ \text { Dirección: } & \text { Joel Grey } \\ \text { Elenco: } & \text { Steven Skybell, Jackie Hoffman, Jennifer Babiak, Joanne } \\ & \text { Borts, Michael Einav, Lisa Fishman, Kirk Geritano, Abby } \\ & \text { Goldfarb, Samantha Hahn, Cameron Johnson, John Giesige, } \\ & \text { Ben Liebert, Moshe Lobel, Stephanie Lynne Mason, Evan } \\ & \text { Mayer, Rosie Jo Neddy, Raquel Nobile, Jonathan Quigley, } \\ & \text { Nick Raynor, Bruce Sabath, Kayleen Seidl, Drew Seigla, } \\ & \text { Adam B. Shapiro, Jodi Snyder, James Monroe Števko, Ron } \\ & \text { Tal, Lauren Jeanne Thomas, Bobby Underwood, Mikhl } \\ & \text { Yashinsky, and Rachel Zatcoff. }\end{array}$




\section{Dibliografía}

" Caplan, D. (2018). Yiddish Empire: The Vilna Troupe, Jewish Theater, and the Art of Itinerancy. Ann Arbor: University of Michigan Press.

"Isenberg, B. (2014). Tradition!: the highly improbable, ultimately triumphant Broadway-to-Hollywood story of Fiddler on the Roof, the world's most beloved musical. Nueva York: St. Martin's Press.

»Nahshon, E. (2016). New York's Yiddish Theater. From the Bowery to Broadway. New York: Columbia University Press.

"Sandrow, N. (1996). Vagabond Stars: A World History of Yiddish Theater. Syracuse: Syracuse University Press.

"Solomon, A. (2013). Wonder of Wonders: A Cultural History of Fiddler on the Roof, Nueva York: Metropolitan Books. 